2019, Vol. 6, No. 1, 84-94.

DOI: https://doi.org/10.17979/reipe.2019.6.1.5355

\title{
Género y liderazgo en cargos de gestión y dirección
}

\section{Gender and leadership in management positions}

\author{
María Dolores Castro (D*, Ana M Porto-Castro (D**, M Josefa Mosteiro-García (D)**, Enelina María Gerpe (iD** \\ * Universidad de Vigo; **Universidad de Santiago de Compostela
}

\begin{abstract}
Resumen
La presencia de las mujeres en el mercado laboral es una realidad hoy en día, no obstante, el acceso a los puestos de responsabilidad y poder, espacios donde podrían ejercer su liderazgo, son todavía una barrera insalvable para ellas. Este trabajo tuvo como objetivo conocer si, en un sector como el bancario, existen diferencias en función del sexo y el cargo de gestión y dirección en lo que respecta a variables de carácter personal y organizacional. Para recopilar dicha información se elaboró una hoja de registro, que fue remitida a todas las entidades bancarias de la Comunidad Autónoma de Galicia, a la que contestaron 448 empleados/as: 315 hombres (70.3\%) y 133 mujeres (29.7\%). Los resultados obtenidos revelaron que tanto directoras como subdirectoras son más jóvenes que sus compañeros directores y subdirectores y tienen un nivel de formación más alto; por el contrario, su trayectoria laboral en el sector de la banca es más corta, su antigüedad en la dirección o subdirección menor y su categoría profesional inferior a la de directores y subdirectores y, en consecuencia, los salarios que perciben menores. Finalmente, en las oficinas, las directoras tienen un número de trabajadores/as a su cargo igual que los directores, pero este número se reduce en el caso de las subdirectoras frente a los subdirectores.
\end{abstract}

Palabras clave: género, liderazgo, gestión, dirección

\begin{abstract}
The presence of women in the working market is a reality today, however, the access to positions of responsibility and power, spaces where they could exercise their leadership, are still an insurmountable barrier for them. In this work, the objective was to know if there are differences according to gender and the position of management and direction with regard to variables of a personal and organizational nature in a sector such as banking. To collect this information, a registration form was drawn up, which was sent to all banking entities in the Autonomous Community of Galicia, to which 448 employees replied: 315 men (70.3\%) and 133 women (29.7\%). The results obtained revealed that both female directors and sub-directors are younger than their fellow
\end{abstract}

María Dolores Castro (iD): Departamento de Didáctica, Organización Escolar y Métodos de Investigación, Facultad de Ciencias de la Educación, Universidad de Vigo, Campus As Lagoas, 32004 Ourense, España.

Ana Ma Porto-Castro (D), Ma Josefa Mosteiro-García (D) Enelina María Gerpe (D): Departamento de Pedagogía y Didáctica, Facultad de Ciencias de la Educacion, Universidad de Santiago de Compostela, Campus Vida: Rúa Prof. Vicente Fráiz Andón, s/n., 15782 Santiago de Compostela, España.

Correspondencia relativa a este artículo: Ana $\mathrm{M}^{\mathrm{a}}$ Porto-Castro - anamaria.porto@usc.es 


\begin{abstract}
directors and assistant directors and have a higher level of education; On the other hand, their work experience in the banking sector is shorter, their seniority in the junior management or subdirector and their professional status lower than that of directors and assistant directors and, consequently, the salaries received are minors. Finally, in the offices, the number of workers in charge of a female director is the same as that of a male director, but that number is reduced when it is they who occupy the sub-directorate in front of their male colleagues.
\end{abstract}

Keywords: gender, leadership, management, direction

Los últimos datos publicados por el Ministerio de Educación, Cultura y Deporte (MECD, 2017) relativos a la Estadística de Estudiantes Universitarios correspondientes al curso académico 2016-2017 señalan que el porcentaje de mujeres con estudios universitarios es más elevado que el de los hombres. Las mujeres representan el 54.7\% del alumnado de Grado y también son mayoría las que cursan estudios de Máster (54.36\%) y Doctorado $(50.33 \%)$.

El número de universitarias egresadas es superior al de universitarios, tanto en los estudios de Grado (58.35\% frente al $41.63 \%$ ) como de Máster (57\% y 42.99\%). Así mismo, a excepción del área de Ingeniería y Arquitectura, en la que el número de mujeres tituladas no alcanza el 30\%, en las restantes ramas de conocimiento (Ciencias Sociales y Jurídicas, Artes y Humanidades, Ciencias de la Salud y Ciencias), el porcentaje de alumnas que finalizan sus estudios universitarios está muy próximo o es superior al $50 \%$.

Estas cifras no se ven del todo reflejadas en el mercado laboral. Según datos del Instituto Nacional de Estadística (INE, 2018), en el año 2017 la tasa de empleo de las mujeres con estudios universitarios era del 76.1\% y la de los hombres del $83.3 \%$, confirmando estos valores la existencia de una brecha de género de 7.2 puntos entre ambos sexos.

A pesar de la elevada formación de las mujeres y de una presencia cada vez mayor en el mercado laboral, el acceso a puestos de responsabilidad y poder parece todavía una barrera insalvable para ellas. Sirva como ejemplo que, en el año 2016, el porcentaje de mujeres en el conjunto de Consejos de Administración de las empresas que formaban parte del Ibex-35 era del 19.1\% (INE, 2018).

La escasa presencia de las mujeres en la cúpula de poder es también limitada en las direcciones ejecutivas de las sociedades mercantiles (Adams, Haan, Terjesen y Ees, 2015; Berenguer, Giráldez y Cardone-Riportella, 2016). En España, según Mateos de Cabo (2017), el escaso avance de las mujeres en los consejos de dirección respecto a otros países como Francia, los Países Bajos o Italia, es debido a la ausencia de sanciones de la Ley de cuotas, la debilidad de complementos institucionales y la falta de iniciativas púbicas para lograr el cambio.

El sector en el que se enmarca el presente estudio, el bancario, comparte una estructura organizacional mecanicista, un sistema de normas y procedimientos altamente prescritos y formalizados, una delimitación clara de los diferentes niveles de poder y también una cultura organizacional muy arraigada en valores masculinos y compuesta mayoritariamente por hombres.

Según un estudio elaborado por el Financial Times en 2016, en el que se analizaron los 50 bancos más grandes del mundo, solo un $25.5 \%$ de mujeres ocupaban las cúpulas de las entidades bancarias y un $39 \%$ desempeñaban cargos intermedios, a pesar de que, en cifras totales, el 58\% del personal empleado en la banca es femenino.

En esta línea, Kilic (2015) comprobó como las mujeres representan tan sólo el 7.96\% de los consejos de dirección de la banca turca. Robles-Elorza, San-José y Urionabarrenetxea (2018), por su parte, constataron que las mujeres ejercen el cargo de directora ejecutiva o directora general en un $17.1 \%$ de las empresas vascas.

Otros autores como Palomo-Zurdo, Gutiérrez-Fernández y Fernández-Torres (2017), comprobaron que en las cooperativas de crédito españolas el género influye en la rentabilidad de estas empresas y varía en función del ciclo económico. 
En opinión de Mateos de Cabo, Gimeno y Nieto (2012) la situación de las mujeres en el sector bancario de la Unión Europea evidencia tres factores que explican su escasa presencia en los puestos de dirección. Por un lado, hay bancos que prefieren una junta directiva de amigos para evitar la contratación de mujeres directoras; por otro, las mujeres son excluidas de los consejos o juntas directivas de bancos con alto riesgo y, por último, los bancos situados en mercados muy dinámicos y competitivos tienen una mayor presencia de mujeres en sus consejos.

En este sector existe además una clara brecha salarial por género. De acuerdo con los últimos datos del Instituto Nacional de Estadística (INE, 2018) recogidos en la Encuesta Anual de Estructura Salarial, las mujeres ganan de media un $23 \%$ menos que los hombres.

La importancia de estos datos es clara a la hora de analizar los puestos laborales que ocupan las mujeres y su liderazgo. Las mujeres están altamente cualificadas, pero siguen teniendo menor poder, esto es, continúan sin asumir la dirección y el liderazgo en las organizaciones, especialmente en aquellas con un marcado sesgo masculinizado como es el caso de las bancarias.

El liderazgo, entendido como la actividad por la que una persona influye en la conducta, creencias y sentimientos de otros miembros del grupo en una dirección intencionada (Sánchez, 1998), es un tema de gran interés en el ámbito organizacional y una de las variables más estudiadas debido a su repercusión en el desempeño laboral y su relación con la satisfacción o el rendimiento.

La diversidad de género en el liderazgo es necesaria en las organizaciones y así lo ponen de manifiesto autores como Droms y Sheryl-Ann (2015) para quienes el liderazgo femenino tiene un impacto positivo sobre el rendimiento y la sostenibilidad financiera de las corporaciones.

En esta misma línea, la investigación de Burke y Collins (2001) confirma que las habilidades directivas de las mujeres favorecen el desarrollo de las personas y la capacidad de comunicación entre los/as trabajadores/as.

Por su parte, el estudio de García-Meca, García-Sánchez y Martínez-Ferrero (2015) con una muestra de 159 bancos de nueve países, pone de manifiesto que las mujeres directivas están altamente cualificadas y tienen características únicas que crean un valor añadido en los bancos, además de que su presencia en los consejos de administración de los bancos mejora su gobernanza. Por su parte, Cuadrado (2014) señala que los estilos de liderazgo "femeninos" serían los que obstaculizan el acceso da la mujer a funciones de dirección.

Molero (2009), por su parte, subraya que el liderazgo eficaz no está ya asociado únicamente a características típicamente masculinas, sino que cada vez se asocia más a características propias del estereotipo femenino, algo que se observa cuando se estudia cuáles son las características que debe tener un directivo o directiva para tener éxito, y cuando los empleados y empleadas evalúan a sus jefes y jefas.

En suma, tal y como indica Cuadrado (2011):

...a pesar de los avances logrados, los puestos de liderazgo continúan estando ocupados mayoritariamente por hombres, debido a los complejos mecanismos que dificultan el acceso de las mujeres. La naturaleza de estos mecanismos es eminentemente psicosocial, por lo que las investigaciones desde esta perspectiva tienen importantes implicaciones para el futuro de las mujeres que pretenden alcanzar el liderazgo en condiciones igualitarias a las de los hombres (p. 293).

Este trabajo se centra en comprobar la existencia de diferencias en función del sexo y el cargo que ocupan empleados y empleadas del sector bancario de la Comunidad Autónoma de Galicia en lo que respecta a algunas variables de carácter personal y de la organización.

Más concretamente, se pretende estudiar si hay diferencias entre directores y directoras y subdirectores y subdirectoras en las siguientes variables: edad, nivel de estudios, puesto que ocupa en la actualidad, antigüedad en la empresa, categoría profesional, antigüedad en el puesto y total de personas trabajando a su cargo en la oficina. 


\section{Método}

\section{Participantes}

En el estudio participan un total de 448 empleados y empleadas de banca, 315 hombres (70.3\%) y 133 mujeres (29.7\%), de edades comprendidas entre los 30 y 60 años y que ocupan puestos de dirección (42.6\% hombres y $8 \%$ mujeres) o subdirección (27.5\% hombres y $21.9 \%$ mujeres) en un total de 226 oficinas bancarias distribuidas geográficamente entre las provincias de A Coruña (45.5\%), Lugo (14.7\%), Orense (12.5\%) y Pontevedra (27.2\%) en la Comunidad Autónoma de Galicia.

\section{Instrumento}

Para la recogida de información se elaboró una hoja de registro en la que se solicitaba a las entidades bancarias de la Comunidad Autónoma de Galicia información referida a variables de carácter personal como el sexo, la edad y el nivel de estudios de los/as trabajadores/as de la empresa y variables referidas a la propia organización como las siguientes: la antigüedad, la categoría profesional, el puesto actual, la antigüedad en el puesto actual y el total de personas trabajando en cada oficina. El total de entidades que respondieron a esta solicitud fue de 226, esto es, el $20 \%$ de oficinas.

\section{Análisis de datos}

Se llevó a cabo, en primer lugar, un análisis descriptivo de las variables objeto de estudio. Posteriormente, se aplicó la prueba de $\chi^{2}$ para comprobar la existencia de diferencias estadísticamente significativas en las variables objeto de estudio, entre mujeres y hombres que ocupan puestos de dirección y subdirección. Se acordó un nivel alfa de $\mathrm{p} \leq .05$ para la interpretación de la significación estadística y se empleó el paquete informático IBM SPSS Statistics versión 24 para la realización de estas pruebas.

\section{Resultados}

En primer lugar, se presentan los datos descriptivos y de comparación para las variables personales y de la organización referidos al puesto de dirección y, en segundo lugar, los que tienen que ver con el puesto de subdirección.

\section{Descriptivos y diferencias en función del sexo y el cargo de dirección en las variables personales y de la organización}

\section{Variables personales}

La media de edad de los/as directores/as es de $43.31(D T=9.23)$. Por lo que se refiere al grupo de varones la media se sitúa un punto por encima ( $M=$ 44.34, $D T=9.39$ ), mientras que en el caso de las directoras está seis puntos por debajo $(M=37.86, D T$ = 5.90). Si se analiza la distribución por tramos de edad (Tabla 1), se observa que la mayoría de los directores se sitúan entre los 41 y 50 años (34\%), mientras que más de la mitad de las directoras (58.3\%) tienen edades comprendidas entre 30 y 40 años.

La mayoría de los/as directores/as son licenciados/as o tienen estudios secundarios o de FP II. Si se tiene en cuenta el sexo, se observa que el $69.4 \%$ de las directoras son licenciadas frente al $37.2 \%$ de los directores, y tan sólo el $25 \%$ de ellas no cursaron estudios universitarios; el porcentaje de directores que no ha realizado estudios universitarios es más elevado (45.8\%) (Tabla 2).

Tabla 1

Porcentajes de directores/as por tramos de edad y sexo

\begin{tabular}{lcc}
\hline Edad & Directoras (\%) & Directores (\%) \\
\hline Menos de 30 años & 8.3 & 7.9 \\
De 30 a 40 años & 58.3 & 27.7 \\
De 41 a 50 años & 27.8 & 34.0 \\
De 51 a 60 años & 5.6 & 30.4 \\
\hline
\end{tabular}


Tabla 2

Porcentajes de directores/as por nivel de estudios y sexo

\begin{tabular}{lcc}
\hline Nivel de estudios & Directoras (\%) & Directores (\%) \\
\hline Primaria/FPI & 0 & 9.6 \\
Secundaria/FPII & 25.0 & 36.2 \\
Diplomatura & 5.6 & 17.0 \\
Licenciatura & 69.4 & 37.2 \\
\hline
\end{tabular}

Si se comparan directores y directoras en las variables edad y estudios cursados, se observa la existencia de diferencias estadísticamente significativas (Tabla 3), lo que pone de manifiesto que las directoras son más jóvenes y tienen un mayor nivel de formación.

Tabla 3

Diferencias en función del sexo en las variables personales

\begin{tabular}{|c|c|c|c|c|}
\hline & & $\begin{array}{c}\text { Directores } \\
(n=315) \\
f(\%)\end{array}$ & $\begin{array}{c}\text { Directoras } \\
(n=133) \\
f(\%)\end{array}$ & $\chi^{2}$ \\
\hline \multirow{4}{*}{ Edad } & Menos de 30 & $15(7.9)$ & $3(8.3)$ & \multirow{4}{*}{$16.114 * *$} \\
\hline & De 30 a 40 años & $53(27.7)$ & $21(58.3)$ & \\
\hline & De 41 a 50 años & $65(34.0)$ & $10(27.8)$ & \\
\hline & De 51 a 60 años & $58(30.4)$ & $2(5.6)$ & \\
\hline \multirow{4}{*}{ Estudios } & Primarios/FPI & $18(9.6)$ & $0(0)$ & \multirow{4}{*}{$14.552 *$} \\
\hline & Secundarios/FPII & $68(36.2)$ & $9(25.0)$ & \\
\hline & Diplomatura & $32(17.0)$ & $2(5.6)$ & \\
\hline & Licenciatura & $70(37.2)$ & $25(69.4)$ & \\
\hline
\end{tabular}

\section{Variables de la organización}

La antiguiedad media en la empresa de los/as directores/as del estudio es de 19.45 años $(D T=$ 11.78). En términos medios, las directoras presentan una trayectoria más corta en el tiempo (13.58 años, $D T=7.05)$ que la de sus homólogos masculinos (20.55 años, $D T=12.17)$.

Si se analiza la distribución de los/as directores/as por tramos de antiguiedad (Tabla 4), se observa que más de la mitad de las directoras (62.9\%) trabajan en la empresa desde hace menos de 10 años, frente a un $31.1 \%$ de directores. Por el contrario, la mayoría de sus compañeros varones, concretamente un $59.6 \%$, tiene una antigüedad en la empresa de 15 años o más, frente a un $37.1 \%$ de las directoras. Así pues, en conjunto, el grupo de directores tiene una mayor experiencia al estar trabajando en la empresa un número superior de años que las directoras.

Tabla 4

Distribución de directores/as por tramos de antigüedad en la empresa y sexo

\begin{tabular}{lcc}
\hline Antigüedad empresa & Directoras (\%) & Directores (\%) \\
\hline Menos de 5 años & 0 & 0.5 \\
De 5 a 9 años & 40.0 & 30.6 \\
De 10 a 14 años & 22.9 & 9.3 \\
De 15 a 19 años & 17.1 & 7.7 \\
20 años o más & 20.0 & 51.9 \\
\hline
\end{tabular}

La categoría profesional de las directoras no alcanza el nivel V en un $36.2 \%$ de los casos y solo el $2.8 \%$ de ellas logra un nivel III o inferior, frente al $67.8 \%$ y $12.6 \%$ de directores respectivamente (Tabla 5). Estos datos son de interés pues los niveles más bajos de esta categoría profesional implican unos ingresos más elevados que los de los niveles profesionales más altos.

Tabla 5

Distribución de directores/as por categoría profesional y sexo

\begin{tabular}{lcc}
\hline Categoría profesional & Directoras (\%) & Directores (\%) \\
\hline Nivel III o inferior & 2.8 & 12.6 \\
Nivel IV & 5.6 & 18.9 \\
Nivel V & 27.8 & 36.3 \\
Nivel VI & 58.3 & 36.3 \\
Nivel VII & 5.6 & 0.5 \\
\hline
\end{tabular}

La antigüedad media en el puesto que desempeñan actualmente los y las directores/as es de 7.15 años (DT $=$ 5.96). Las mujeres ocupan su puesto como directoras, por término medio, aproximadamente desde hace algo más de tres años $(M=3.73, D T=3.2)$, mientras que los directores son más antiguos en el puesto actual, sobrepasando la media de siete años de antigüedad en el mismo $(M=7.78, D T=6.15)$.

En conjunto, la mayoría de los directores $(44.8 \%)$ y las directoras (79.4\%) tiene una antigüedad en el puesto de menos de 5 años. El $94.1 \%$ de las directoras ocupa este puesto desde hace menos de 10 años y sólo el 5.9\% más de 10 años. En el caso de sus compañeros varones, el 32.8\% tiene una antigüedad en el puesto superior a 10 años y el $67.2 \%$ de menos de 10 años (Tabla 6). 
Tabla 6

Distribución de directores/as por tramos de antigüedad en el puesto actual y sexo

\begin{tabular}{lcc}
\hline Antigüedad en el puesto & Directoras (\%) & Directores (\%) \\
\hline Menos de 5 años & 79.4 & 44.8 \\
De 5 a 9 años & 14.7 & 22.4 \\
De 10 a 14 años & 5.9 & 16.4 \\
De 15 a 19 años & 0 & 11.5 \\
20 años o más & 0 & 4.9 \\
\hline
\end{tabular}

Cuando se analiza el número concreto de personas trabajando en las distintas oficinas y el sexo de quienes están al frente de las mismas en el puesto de dirección, se observa que las mujeres lideran equipos de trabajo prácticamente iguales que los de sus compañeros varones, con valores entre el $40 \%$ y $50 \%$ (Tabla 7).

Tabla 7

Distribución de directores/as por total de personas trabajando en la oficina y sexo

\begin{tabular}{lcc}
\hline Tamaño equipos & Directoras (\%) & Directores (\%) \\
\hline De 1 a 3 personas & 41.7 & 50.3 \\
De 4 a 6 personas & 47.2 & 37.2 \\
De 7 a 9 personas & 8.3 & 7.9 \\
10 o más personas & 2.8 & 4.7 \\
\hline
\end{tabular}

En la Tabla 8 se puede observar la existencia de diferencias estadísticamente significativas al comparar directores y directoras en las variables de la organización como la antigüedad en la empresa $\left(\chi^{2}=\right.$ $13.347 ; \mathrm{p}=.004)$, la categoría profesional $\left(\chi^{2}=\right.$ $15.477 ; \mathrm{p}=.004)$ y la antigüedad en el puesto actual $\left(\chi^{2}=15.359 ; \mathrm{p}=.004\right)$, pero no en el caso de la variable total de personas trabajando en la oficina $\left(\chi^{2}\right.$ $=1.513 ; \mathrm{p}=.679)$.

Por tanto, las mujeres llevan menos años trabajando en la empresa que los hombres. Las diferencias nuevamente son a favor de los hombres en la antiguiedad en el puesto, es decir, ellos ostentan la dirección desde hace más años. Finalmente, respecto a la categoría profesional, son los hombres los que se sitúan en los niveles III e inferiores que se corresponden a los de mayor categoría profesional y más elevada remuneración económica.
Tabla 8

Diferencias en función del sexo y el cargo de director/a en las variables de la organización

\begin{tabular}{|c|c|c|c|c|}
\hline & & $\begin{array}{c}\text { Directores } \\
(n=315) \\
f(\%) \\
\end{array}$ & $\begin{array}{c}\text { Directoras } \\
(n=133) \\
f(\%) \\
\end{array}$ & $\chi^{2}$ \\
\hline \multirow{4}{*}{$\begin{array}{l}\text { Antigüedad en } \\
\text { empresa } \\
\text { (años) }\end{array}$} & De 5 a 9 & $15(44.1)$ & $5(50)$ & \multirow{4}{*}{$13.347 *$} \\
\hline & De 10 a 14 & $1(2.9)$ & $4(40)$ & \\
\hline & De 15 a 19 & $5(14.7)$ & $1(10.0)$ & \\
\hline & 20 o más & $13(38.2)$ & $0(0)$ & \\
\hline \multirow{5}{*}{$\begin{array}{l}\text { Categoría } \\
\text { profesional }\end{array}$} & Nivel III o < & $24(12.6)$ & $1(2.8)$ & \multirow{5}{*}{$15.477^{\prime}$} \\
\hline & Nivel IV & $36(18.9)$ & $2(5.6)$ & \\
\hline & Nivel V & $60(36.3)$ & $10(27.8)$ & \\
\hline & Nivel VI & $69(36.3)$ & $21(58.3)$ & \\
\hline & Nivel VII & $1(0.5)$ & $2(5.6)$ & \\
\hline \multirow{5}{*}{$\begin{array}{l}\text { Antigüedad en } \\
\text { puesto actual } \\
\text { (años) }\end{array}$} & Menos de 5 & $82(44.8)$ & $27(79.4)$ & \multirow{5}{*}{$15.359^{*}$} \\
\hline & De 5 a 9 & $41(22.4)$ & $5(14.7)$ & \\
\hline & De 10 a 14 & $30(16.4)$ & $2(5.9)$ & \\
\hline & De 15 a 19 & $21(11.5)$ & $0(0)$ & \\
\hline & 20 o más & $9(4.9)$ & $0(0)$ & \\
\hline $\begin{array}{l}\text { Total personas } \\
\text { trabajando en } \\
\text { oficina }\end{array}$ & De 1 a 3 & $96(50.3)$ & $15(41.7)$ & 1.513 \\
\hline
\end{tabular}

\section{Diferencias en función del sexo y el cargo de subdirección en las variables personales y de la organización}

\section{Variables personales}

La edad media de los/as trabajadores/as que ocupan el cargo de subdirección es de 42 años, aproximadamente $(M=42.11, D T=9.40)$. El grupo de subdirectores es algo mayor que la media total $(M$ $=45.85$ años, $D T=9.55)$ y el de las subdirectoras algo más joven $(M=37.41$ años, $D T=6.74)$.

A medida que se incrementa el tramo de edad, la presencia de mujeres subdirectoras disminuye. Así, en la Tabla 9 se puede observar cómo un $8.2 \%$ de las subdirectoras tiene menos de 30 años frente al $5.7 \%$ de hombres. Las diferencias se incrementan en el tramo de edad de 30 a 40 años, en el que se sitúa un $65.3 \%$ de subdirectoras y tan sólo un $26 \%$ de subdirectores. A partir de los 40 años los datos se invierten, aumentando el número de subdirectores (38.2\%) y disminuyendo el de subdirectoras (7.1\%).

Por otro lado, los datos recogidos en la Tabla 10 muestran que la mayoría de los subdirectores y de las subdirectoras tienen estudios universitarios de 
licenciatura y estudios secundarios o de FP II. Si se tiene en cuenta el sexo, se observa que un $56.7 \%$ de las subdirectoras son licenciadas y un $20.6 \%$ diplomadas, frente a un $32.8 \%$ y un $16.8 \%$ de subdirectores que poseen esos niveles de formación respectivamente. Estos datos se invierten en los niveles más bajos, pues el porcentaje de subdirectores con estudios primarios o de FPI (15.1\%) y de estudios secundarios o FPII (35.3\%) es más elevado que el obtenido por las subdirectoras $(3.1 \%$ y $\quad 19.6 \%$ respectivamente).

Tabla 9

Distribución de subdirectores/as por tramos de edad y sexo

\begin{tabular}{lcc}
\hline Edad (años) & Subdirectoras (\%) & Subdirectores (\%) \\
\hline Menos de 30 & 8.2 & 5.7 \\
De 30 a 40 & 65.3 & 26.0 \\
De 41 a 50 & 19.4 & 30.1 \\
De 51 a 60 & 7.1 & 38.2 \\
\hline
\end{tabular}

Tabla 10

Porcentajes de subdirectores/as por nivel de estudios y sexo

\begin{tabular}{lcc}
\hline Nivel de estudios & Subdirectoras (\%) & Subdirectores (\%) \\
\hline Primaria/FPI & 3.1 & 15.1 \\
Secundaria/FPII & 19.6 & 35.3 \\
Diplomatura & 20.6 & 16.8 \\
Licenciatura & 56.7 & 32.8 \\
\hline
\end{tabular}

Tabla 11

Diferencias en función del sexo y el cargo de subdirector/a en las variables personales

\begin{tabular}{|c|c|c|c|c|}
\hline & & $\begin{array}{c}\text { Subdirectores } \\
(n=315) \\
f(\%)\end{array}$ & $\begin{array}{c}\text { Subdirectoras } \\
(n=133) \\
f(\%)\end{array}$ & $\chi^{2}$ \\
\hline \multirow{4}{*}{ Edad } & Menos de 30 & $7(5.7)$ & $8(8.2)$ & \multirow{4}{*}{$43.882 *$} \\
\hline & De 30 a 40 años & $32(26)$ & $64(65.3)$ & \\
\hline & De 41 a 50 años & $37(30.1)$ & $19(19.4)$ & \\
\hline & De 51 a 60 años & $47(38.2)$ & $7(7.1)$ & \\
\hline \multirow{5}{*}{\multicolumn{2}{|c|}{$\begin{array}{c}\text { Primarios/FPI } \\
\text { Secundarios/FPII } \\
\text { Estudios Diplomatura } \\
\text { Licenciatura } \\
\text { Máster }\end{array}$}} & $18(15.1)$ & $3(3.1)$ & \multirow{5}{*}{20.077} \\
\hline & & $42(35.3)$ & 19 (19.6) & \\
\hline & & $20(16.8)$ & $20(20.6)$ & \\
\hline & & $39(32.8)$ & $55(56.7)$ & \\
\hline & & $0(0)$ & $0(0)$ & \\
\hline
\end{tabular}

En la Tabla 11, se constatan diferencias estadísticamente significativas entre subdirectoras y subdirectores en lo que respecta a la variable edad $\left(\chi^{2}\right.$ $=43.882 ; p<.001)$, destacando que, con el aumento en edad, disminuye de modo considerable el número de mujeres que ocupan el puesto de subdirectoras.

Por lo que respecta a la variable nivel de estudios $\left(\chi^{2}=20.077 ; p<.001\right)$ las diferencias observadas entre subdirectoras y subdirectores son también estadísticamente significativas, siendo las mujeres las que alcanzan un nivel de formación superior al de los hombres.

\section{Variables de la organización}

La antiguiedad media en la empresa de los/as subdirectores/as que participan en el estudio es de 19.04 años $(D T=12.37)$. Ellas tienen menos antigüedad en la empresa $(M=13.09$ años, $D T=8.91)$ que ellos $(M=23.78$ años, $D T=12.72)$.

Un $52.1 \%$ de las subdirectoras llevan de 5 a 9 años trabajando en la empresa, mientras que, por el contrario, algo más de la mitad (59.3\%) de los hombres hace más de 20 años (Tabla 12).

Tabla 12

Distribución de subdirectores/as por tramos de antigüedad en el puesto actual y sexo

\begin{tabular}{lcc}
\hline $\begin{array}{l}\text { Antigüedad en el } \\
\text { puesto }\end{array}$ & $\begin{array}{c}\text { Subdirectoras } \\
(\%)\end{array}$ & $\begin{array}{c}\text { Subdirectores } \\
(\%)\end{array}$ \\
\hline Menos de 5 años & 1.0 & 1.7 \\
De 5 a 9 años & 52.1 & 21.2 \\
De 10 a 14 años & 16.7 & 0.1 \\
De 15 a 19 años & 10.4 & 12.7 \\
20 años o más & 19.8 & 59.3 \\
\hline
\end{tabular}

La categoría profesional de la mayoría de los subdirectores y subdirectoras se sitúa en el nivel $\mathrm{V}$ o superior. En los niveles V e inferiores hay un mayor número de subdirectores (39\%) que subdirectoras (8.1\%). Por el contrario, el porcentaje de subdirectoras con niveles VI y VII es mayor, alcanzando porcentajes del $89.8 \%$, lejos del $60.2 \%$ que corresponde a los subdirectores (Tabla 13).

$\mathrm{La}$ antiguiedad media de quienes trabajan actualmente en el puesto de subdirección es de 4.69 años $(D T=2.09)$. Por término medio las mujeres 
tienen una antigüedad como subdirectoras de 4.10 años $(D T=2.64)$, mientras los subdirectores ocupan este cargo desde hace 5.16 años $(D T=3.03)$.

Tabla 13

Distribución de subdirectores/as por categoría profesional y sexo

\begin{tabular}{lcc}
\hline Categoría & Subdirectoras (\%) & Subdirectores (\%) \\
\hline Nivel III o inferior & 1.0 & 4.1 \\
Nivel IV & 2.0 & 8.9 \\
Nivel V & 5.1 & 26.0 \\
Nivel VI & 37.8 & 31.7 \\
Nivel VII & 52.0 & 28.5 \\
\hline
\end{tabular}

En general, las personas participantes ocupan la subdirección desde hace poco tiempo, pues ninguno/a tiene una antigüedad como subdirector/a de más de 10 años. Si se tiene en cuenta el sexo, se observa que hay un porcentaje más elevado de subdirectoras (62.2\%) que subdirectores $(44.7 \%)$ con menos de 5 años de antigüedad en el puesto, valores que se invierten en el tramo de antigüedad de 5 a 9 años, en el que se sitúa un $55.3 \%$ de subdirectores y un $37.8 \%$ de subdirectoras.

Como recoge la Tabla 14, un 52\% de subdirectores y un $44.9 \%$ de subdirectoras tienen a su cargo entre 1 y 3 trabajadores/as. Estos porcentajes se invierten cuando el número de trabajadores/as en la oficina es superior a 4, situación en la que ostenta la subdirección un $47.9 \%$ de hombres y un $55.1 \%$ de mujeres.

Tabla 14

Distribución de subdirectores/as por total de personas trabajando en la oficina y sexo

\begin{tabular}{lcc}
\hline Tamaño equipos & Subdirectoras (\%) & Subdirectores (\%) \\
\hline De 1 a 3 & 44.9 & 52.0 \\
De 4 a 6 & 45.9 & 33.3 \\
De 7 a 9 & 8.2 & 6.5 \\
10 o más & 1.0 & 8.1 \\
\hline
\end{tabular}

En la Tabla 15 se constatan diferencias estadísticamente significativas entre subdirectoras y subdirectores en lo que respecta a las variables antigüedad en la empresa $\left(\chi^{2}=12.473 ; p=.006\right)$ y antigüedad en el puesto actual $\left(\chi^{2}=6.721 ; p=.010\right)$. Estas diferencias muestran que las subdirectoras ocupan su cargo desde hace menos tiempo que los hombres, y trabajan desde hace menos años en la empresa.

Así mismo, se constata la existencia de diferencias estadísticamente significativas en lo que respecta a las variables categoría profesional $\left(\chi^{2}=29.512 ; p<.001\right)$ $\mathrm{y}$ número total de personas trabajando en la oficina $\left(\chi^{2}\right.$ $=8.535 ; p=.036)$. Las diferencias encontradas revelan que las subdirectoras tienen una categoría profesional con más baja remuneración que la de los hombres y que lideran oficinas con un menor número de trabajadores/as.

Tabla 15

Diferencias en función del sexo y el cargo de subdirector/a en las variables de la organización

\begin{tabular}{|c|c|c|c|c|}
\hline & & $\begin{array}{c}\text { Subdirector } \\
\begin{array}{c}(n=315) \\
f(\%)\end{array}\end{array}$ & $\begin{array}{c}\text { ubdirectora } \\
(n=133) \\
f(\%)\end{array}$ & $\chi^{2}$ \\
\hline \multirow{4}{*}{$\begin{array}{l}\text { Antigüedad } \\
\text { empresa } \\
\text { (años) }\end{array}$} & De 5 a 9 & $5(19.2)$ & $11(64.7)$ & \multirow{4}{*}{$12.473 * *$} \\
\hline & De 10 a 14 & $1(3.8)$ & $2(11.8)$ & \\
\hline & De 15 a 19 & $5(19.2)$ & $2(11.8)$ & \\
\hline & 20 o más & $15(57.7)$ & $2(11.8)$ & \\
\hline \multirow{5}{*}{$\begin{array}{l}\text { Categoría } \\
\text { profesional }\end{array}$} & Nivel III o < & $5(4.1)$ & $1(1)$ & \multirow{5}{*}{$29.512 * * *$} \\
\hline & Nivel IV & $11(8.9)$ & $2(2)$ & \\
\hline & Nivel V & $32(26)$ & $5(5.1)$ & \\
\hline & Nivel VI & 39 (31.7) & 37 (37.8) & \\
\hline & Nivel VII & $35(28.5)$ & $51(52)$ & \\
\hline \multirow{3}{*}{$\begin{array}{l}\text { Antigüedad } \\
\text { puesto } \\
\text { actual } \\
\text { (años) } \\
\end{array}$} & Menos de 5 & $55(44.7)$ & $61(62.2)$ & \multirow{3}{*}{$6.721 *$} \\
\hline & De 5 a 9 & $68(55.3)$ & 37 (37.8) & \\
\hline & De 10 a 14 & $0(0)$ & $0(0)$ & \\
\hline \multirow{4}{*}{$\begin{array}{l}\text { Total } \\
\text { personas } \\
\text { trabajando } \\
\text { en oficina }\end{array}$} & De 1 a 3 & $64(52)$ & 44 (44.9) & \multirow{4}{*}{$8.535^{*}$} \\
\hline & De 4 a 6 & $41(33.3)$ & 45 (45.9) & \\
\hline & De 7 a 9 & $8(6.5)$ & $8(8.2)$ & \\
\hline & 10 o más & $10(8.1)$ & $1(1)$ & \\
\hline
\end{tabular}

\section{Discusión}

Los resultados expuestos anteriormente ponen de manifiesto que mujeres y hombres comparten un mismo espacio laboral pero no lo hacen de forma equitativa, sobre todo en el acceso a los puestos de responsabilidad y poder (Adams et. al. 2015; Berenguer et al., 2016; Kilic, 2015; Robles-Elorza et. al., 2018). 
Así, los datos analizados reflejan que tanto para el caso de las directoras como de las subdirectoras objeto de estudio, la edad a la que acceden al cargo es, en general, menor que la de sus compañeros varones; en su mayoría trabajan en la empresa y ocupan el cargo de directoras o subdirectoras desde hace menos tiempo que ellos, y aun cuando su nivel de formación es superior, el puesto profesional que ocupan es de menor categoría, resultados que coinciden con los encontrados por Pereda-Pérez, López-Guzmán y González-Santa Cruz (2018) en un estudio sobre habilidades directivas y diferencias de género entre el funcionariado del sector público.

Por lo que se refiere a las diferencias por sexo, los hombres ocupan puestos de trabajo con mayor retribución económica que las mujeres. Así mismo, ellas ocupan puestos directivos o de responsabilidad en un 42,9\% de los casos frente al 55\% de los ocupados por ellos. Estas diferencias hunden sus raíces en mecanismos históricos tales como la socialización familiar y profesional, la educación, las dificultades para conciliar los roles profesionales y familiares, según la etapa del ciclo de vida, y los estereotipos aún vigentes en las organizaciones respecto a la presencia de mujeres en los puestos de alta responsabilidad.

En relación con la edad, si bien los datos reflejan resultados muy semejantes, es destacable la mayor juventud de las mujeres. El predominio de personas en el intervalo de 46-55 años implica una plantilla media madura, que provocará a medio plazo un fuerte proceso de jubilaciones, lo que supondrá, a su vez, un esfuerzo de planificación de recursos humanos muy notable con el fin de proporcionar los efectivos necesarios y adecuados a la empresa.

Por una parte, la incorporación tardía de las mujeres al sector bancario provoca una similitud de representación de este grupo en las edades más tempranas con respecto a los hombres, con una clara merma de presencia femenina a partir de los 45 años. Este hecho también se refleja en la antigüedad dentro de la organización, pues la mayoría de las mujeres que ocupan puestos de dirección o subdirección se incorporaron a la empresa en los últimos 20 años.

Por otra parte, la formación académica de mujeres y hombres muestra tendencias muy diferentes; a medida que ascendemos en el nivel académico, el porcentaje de mujeres mejor formadas aumenta de manera considerable con respecto a sus compañeros varones. Sin embargo, ocupando un mismo cargo, las mujeres perciben menos ingresos que los hombres; en concreto, más de la mitad de las mujeres de la muestra estudiada tienen un nivel VII o superior, que se corresponde con un menor salario.

Finalmente, el número de personas que integra el grupo liderado por las directoras no parece diferir del que corresponde, en las mismas circunstancias, a sus homólogos masculinos; sin embargo, en el caso de las subdirectoras este número es bastante más bajo, en la línea de los resultados encontrados por Robles-Elorza et. al. (2018) quienes comprobaron en su estudio que cuando es una mujer la que está al frente de la dirección ejecutiva de la empresa tiene aproximadamente la mitad de trabajadores/as a su cargo que cuando en la misma posición corporativa está un hombre.

El marco laboral estudiado, el bancario, al igual que otros muchos, sigue siendo un sector masculinizado en el que las mujeres todavía no han podido romper con los estereotipos de género y acceder en igualdad a puestos de responsabilidad y liderazgo.

Este estudio se ha visto condicionado por una serie de limitaciones, entre las que destacan, fundamentalmente, la ausencia de datos sobre determinadas características que pueden reducir la presencia de mujeres en el sector de la banca, como, por ejemplo, las relacionadas con la conciliación y el desarrollo de la carrera profesional, aspectos que se erigen como posibles futuras líneas de investigación. Así mismo, hay que tener en cuenta que se trata de un estudio sectorial, no generalizable al conjunto de actividades empresariales de otros sectores, aunque aporte resultados apreciables para el sector concreto en el que está centrado, el bancario, y que además se sitúa en un territorio específico, el gallego, con sus peculiaridades.

Finalmente, cabe señalar que para el avance en la investigación sobre el liderazgo desde una perspectiva de género sería deseable analizar otros contextos laborales en donde la mayor o menor presencia de mujeres y 
hombres permita, por una parte, el establecimiento de políticas específicas de desarrollo de los recursos humanos, y por otra parte, avanzar en el conocimiento de las diferencias entre mujeres y hombres para determinar las competencias que tienen una mayor capacidad predictiva sobre el liderazgo de los empleados y empleadas.

\section{Referencias}

Adams, R., Haan, J., Terjesen, S., \& Ees, H. (2015). Board diversity: Moving the field forward. Corporate Governance: An International Review, 23(2), 77-82. https://doi.org/10.1111/corg.12106

Berenguer, E., Giráldez, P., \& Cardone-Riportella, C. (2016). Managerial positions of women in Spanish exporting SMEs. Spanish Journal of Finance and Accounting, 45(3), 300-326. https://doi.org/10.1080/02102412. 2016.1168622

Burke, S., \& Collins, K (2001). Gender Differences in Leadership Styles and Management Skills. Women in Management Review, 16, 244-257. https://doi.org/10.1108/09649420110395728

Droms, C., \& Sheryl-Ann, S. (2015). Gender Effects on Perceptions of Individual and Corporate Social Responsibility. Journal of Applied Business and Economics, 17 (3), 63-71. http://www.na-businesspress. com/jabeopen.html

Cuadrado, I. (2011). Liderazgo y discriminación femenina. En F. Molero y J.F. Morales (Coords.), Liderazgo: hecho y ficción (pp. 271-294). Madrid: Alianza Editorial.

Cuadrado, I. (2014). Do women and men adopt different leadership styles?. An analysis of the influence of leadership styles on access to managerial positions. International Journal of Social Psychology, 18 (3), 283307. https://doi.org/10.1174/021347403322470864

Financial Times (25 de noviembre de 2018). Financial Times. Disponible en: http://www.expansion.com/ empresas/banca/2017/04/05/58e3fa8422601d22718b4585.htm

García-Meca, E., García-Sánchez, I-M., \& Martínez-Ferrero, J. (2015). Board diversity and its effects on bank performance: An international analysis. Journal of banking \& Finance, 53, 202-214. https://doi.org/10.1016/j.jbankfin.2014.12.002

Instituto Nacional de Estadística (INE, 2018). Mujeres y hombres en España. Disponible en: http://www.ine.es/ ss/Satellite?c=INEPublicacion_C\&cid=1259924822888\&p=1254735110672\&pagename=ProductosYServici os\%2FPYSLayout\&param1=PYSDetalleGratuitas

Instituto Nacional de Estadística (INE, 2018). Encuesta Anual de Estructura Salarial. Serie 2008-2016. Disponible en: http://www.ine.es/dyngs/INEbase/es/operacion.htm?c=Estadistica_C\&cid=1254736177025\&menu=ultiDa tos\&idp $=1254735976596$

Kilic, M. (2015). The effect of board diversity on the performance of banks: Evidence from Turkey. International Journal of Business and Management, 10 (9), 182-192. https://doi.org/10.5539/ijbm.v10n9p182

Mateos de Cabo, R. (2017). Mujeres y liderazgo empresarial: explorando la brecha de género en la cima. Femeris, 2 (2), 53-80. https://doi.org/10.20318/femeris.2017.3758

Mateos de Cabo, R., Gimeno, R., \& Nieto, M.J. (2012). Gender diversity on European banks` boards of directors: Traces of discrimination. Journal of Business Ethics, 109 (2), 145-162. https://doi.org/10.1007/s10551-011$1112-6$

Ministerio de Educación, Cultura y Deporte (MECD, 2017). Estadística de Estudiantes Universitarios. Curso 2016-2017. Disponible en: http://www.educacionyfp.gob.es/servicios-al-ciudadano-mecd/estadisticas/educa cion/universitaria/estadisticas/alumnado/2016-2017_Rend.html

Molero, F. (2009). Mujer y liderazgo en el siglo XXI: una aproximación psicosocial a los factores que dificultan el acceso de la mujer a los puestos de alta responsabilidad. Madrid: Instituto de la Mujer. Disponible en: http://www.inmujer.gob.es/gl/areasTematicas/estudios/estudioslinea2009/docs/mujerLiderazgo.pdf 
Palomo-Zurdo, R., Gutiérrez-Fernández, M., \& Fernández-Torres, Y. (2017). La cuestión del género en los órganos de gobierno de la banca cooperativa. Revista de Economía Pública, Social y Cooperativa, 89, 137-166. Pereda-Pérez, F. J., López-Guzmán, T., \& González-Santa Cruz, F. (2018). Habilidades directivas y diferencias de género en el sector público: el caso de Córdoba (España). Revista Española de Ciencia Política, 46, 199230. https://doi.org/10.21308/recp.46.09

Robles-Elorza, D., San-José, L., \& Urionabarrenetxea, S. (2018). El género de la dirección ejecutiva: análisis e implicaciones financieras. Revista de Responsabilidad Social de la Empresa, 30, 15-36.

Sánchez, E. (1998). Liderazgo y estilos de dirección. En A. Rodríguez (Eds.), Introducción a la psicología del trabajo y de las organizaciones (pp.153-166). Madrid: Pirámide.

Fecha de recepción: 6 de marzo de 2019.

Fecha de revisión: 21 de junio de 2019.

Fecha de aceptación: 24 de junio de 2019.

Fecha de publicación: 1 de julio de 2019. 